\title{
Elimination of Environmental Effects on Temperature Assessment in Clinical Postoperative Free Flap Monitoring - A Prospective Cohort Study
}

\section{Kraemer Robert ${ }^{1 * \#}$, Lorenzen Johan ${ }^{2 \#}$, Wuerfel Waldemar ${ }^{3}$, Papst Stephan ${ }^{1}$, Busche Marc ${ }^{1}$, Knobloch Karsten ${ }^{1}$ and Vogt Peter M ${ }^{1}$}

${ }^{1}$ Plastic, Hand and Reconstructive Surgery, Hannover Medical School, Carl-Neuberg-Strasse 1, 30625 Hannover, Germany

${ }^{2}$ Department of Nephrology, Hannover Medical School, Germany, Carl-Neuberg-Strasse 1, 30625 Hannover, Germany

${ }^{3}$ Department of Otolaryngology, Hannover Medical School, Germany, Carl-Neuberg-Strasse 1, 30625 Hannover, Germany

\#Both authors contributed equally to this work

\begin{abstract}
Background: Only few studies have currently examined the hemodynamics of free tissue transfer to correlate quantitative results with clinical and technical observations. The aim of this study was to further evaluate the free flap skin island temperature as a potential tool of postoperative flap monitoring as temperature monitoring is more reliable if environmental influences on tissue temperature are eliminated.
\end{abstract}

Hypothesis: Differences between free flap Skin Island and healthy adjacent skin microcirculation correlate to the difference between free flap Skin Island and healthy adjacent skin temperatures.

Methods: ClinicalTrials.gov NCT01235299. Prospective cohort study. 56 free flaps (24 latissimus dorsi flaps, 11 ALT,10 parascapular, 9 DIEP, 1 serratus, 1 VRAM) in 56 patients ( 35 males, age 49 $\pm 14 \mathrm{yrs}$ ) were prospectively monitored from November 2009 to January 2011 using a regular digital infrared surface thermometer simultaneously to microcirculatory assessment using combined Laser-Doppler and photo spectrometry (Oxygen-to-see).

Results: Free flap microcirculatory blood flow was $109 \pm 37$ arbitrary units [AU]. Adjacent skin capillary blood flow was $143 \pm 52 \mathrm{AU}$. Free flap skin island temperature was $95.3 \pm 3.0^{\circ} \mathrm{F}$. Temperature of adjacent skin was $96.6 \pm 1.9^{\circ} \mathrm{F}$. We found a positive correlation between the percentaged difference of free flap and adjacent skin temperatures and percentaged difference of free flap skin island and adjacent skin capillary blood flow $(p=0.001)$.

Conclusion: Our hypothesis was confirmed. A 10\% reduction of free flap skin island temperature compared to adjacent skin indicates a deterioration of flap microcirculation by $50 \%$, if skin temperature of the adjacent skin remains the same. Trends in surface skin island temperature monitoring are found to correlate with microcirculatory blood flow. Eliminating environmental influences on temperature assessment by comparing the temperature of the skin island of the free flap to the temperature of the adjacent healthy skin makes free flap temperature monitoring more reliable in reconstructive microsurgery.

Keywords: Free flap; Monitoring; Temperature; Microcirculation

\section{Introduction}

The time interval between the onset of ischemia and its clinical recognition is of utmost importance in terms of free flap salvage in free flap surgery [1]. Although microsurgical free-tissue transfer has become a reliable technique, between 5 to 25 percent of transferred flaps require surgical revision because of circulatory compromise either on the arterial or venous side [2]. Regardless of the surgeon's experience or the reliability of the chosen free flap, immediate detection of perfusion failure and prompt revision is paramount for flap salvage as ischemic tolerance of the flap tissue is limited to a few hours and irreversible disseminated microvascular thrombi may form during malperfusion [3]. After completion of the microsurgical anastomosis, the success of a free-tissue transfer is dependent on the continuous arterial inflow and venous outflow until the tissue is neovascularized by peripheral ingrowth of vessels [4]. Consecutively, close monitoring of free flap perfusion is mandatory in the postoperative period for early diagnosis of perfusion failure [5].

However, to date no single gold standard exists and research is ongoing evaluating innovative as well as reknown measures of monitoring. Besides the clinical inspection and capillary refill testing, various technical methods have been suggested to be useful in this regard. As a simple method of free flap monitoring, surface temperature recordings have already been demonstrated to be correlated to flap failure clinically [6]
Although absolute cut-off temperatures have already been described below which flaps do not survive, this could not be proven to be predictive in postoperative free flap monitoring as temperature is subject to systemic physiological changes in the patient [7]. After initial enthusiasm for this simple and economic principle of free flap monitoring, several issues mostly concerning environmental factors have been raised such as limited spatial resolution, dependence on room temperature, air flow and flap coverage with dressings, sheets, and blankets, which are thought to limit the practical value of temperature readings for flap monitoring to date [8,9].

Papillion et al. recently demonstrated a trend in free flap temperature

${ }^{*}$ Corresponding author: Robert Kraemer MD, Plastic, Hand and Reconstructive Surgery, Hannover Medical School, Carl-Neuberg-Str. 1, 30625 Hannover, Germany, Tel: 0049-511-532-8864; Fax: 0049-511-532-8890; E-mail: Kraemer.Robert@ mh-hannover.de

Received August 30, 2011; Accepted October 22, 2011; Published October 25 2011

Citation: Kraemer R, Lorenzen J, Wuerfel W, Papst S, Busche M, et al. (2011) Elimination of Environmental Effects on Temperature Assessment in Clinical Postoperative Free Flap Monitoring - A Prospective Cohort Study. Anaplastology S1:001. doi:10.4172/2161-1173.S1-001

Copyright: ( 2011 Kraemer R, et al. This is an open-access article distributed under the terms of the Creative Commons Attribution License, which permits unrestricted use, distribution, and reproduction in any medium, provided the original author and source are credited. 
that correlates with anastomotic thrombosis and eventual flap failure in postoperative free flap temperature monitoring [10]. Chiu et al. published the use of temperature strips to determine free flap skin temperature as an accurate tool for postoperative free flap monitoring [11].

Recently we could demonstrate that free flap skin temperature is closely related to free flap microcirculation applying temperature assessment and non-invasive continuous combined laser Doppler and spectrophotometry in a clinical situation [12]. So far, no other study has examined the microcirculatory hemodynamics of free tissue transfer to correlate with clinical and technical microcirculatory observations. According to our recent study about free flap temperature monitoring, we suggested to compare temperature changes of the transplanted flap in contrast to the temperature of the adjacent skin to the flap in order to eliminate or at least minimize environmental influences on tissue temperature as temperature assessment might be more reliable if free flap skin temperature could be correlated to patient's adjacent healthy skin. Therefore, we hypothesized that differences between free flap and healthy adjacent skin microcirculation correlate to the difference between free flap skin temperature and healthy adjacent skin temperature in order to elucidate the possible elimination of environmental factors in free flap skin temperature monitoring.

\section{Material and Methods}

The study was performed in conformity to the World Medical Association Declaration of Helsinki (June 1964) and subsequent amendments. Throughout this study, there was no further data assessment than regular flap monitoring including documentation of microcirculatory parameters.

\section{Clinical trial registration}

The trial was registered at ClinicalTrials.gov with the identifier number NCT01235299. IRB approval was granted at the local IRB committee.

\section{Reporting standards}

Furthermore, the study is reported according to the STARD-statement, e.g. standards for the reporting of diagnostic accuracy studies [13].

\section{Participants}

\section{Study population and recruitment:}

a) Inclusion criteria: Eligibility criteria were patients suffering from a soft tissue defect at any location of the body receiving free flap soft-tissue defect coverage at the Department of Plastic, Hand and Reconstructive Surgery, Medical School Hannover, Germany. Inclusion criteria were age between 16 and 65 years.

b) Exclusion criteria: Exclusion criteria were soft tissue inflammation or osteomyelitis, peripheral arterial occlusive disease, vasculitis, chronic kidney or liver disease, cardiac dysfunction, arterial hypotension and any type of vasoactive medication, i.e. ß-blockers, calcium channel blockers, nitroglycerin or equal. Furthermore, for standardized temperature assessment all transplanted flaps without a skin component were excluded from the study.

The study population was a consecutive series of participants defined by the aforementioned selection criteria.

Setting and test methods: Regular free flap microcirculatory monitoring at our department features combined Laser-Doppler and photospectrometry (Oxygen-to-see, Lea Medizintechnik, Germany) to determine postoperative free flap micorcirculation. Additionally to the determination of free flap microcirculation, temperature assessment of the free flaps was performed using a regular digital infrared surface thermometer after microcirculatory assessment (Medisana FTD, Germany). Four standardized locations for temperature assessment were determined on each flap and furthermore at four representative locations of healthy adjacent skin to the transplanted free flap. Simultaneously to temperature measurement, microcirculation of the free flaps was assessed at the same distinct locations on the flaps and adjacent skin, respectively, using combined Laser-Doppler and photospectrometry. Microcirculation and temperature was assessed between first and seventh postoperative day. Patients received standard deep venous thrombosis prophylaxis with either 15000IE unfractionated heparin or low risk dosaged low molecular weight heparin as well as $500 \mathrm{mg}$ of aspirin per day, postoperatively.

\section{Test reference methods}

Determination of vital parameters of microcirculation: The determination of hemoglobin and the principle of blood flow measurement are combined in the $\mathrm{O} 2 \mathrm{C}$ system. The optical method for measuring both, blood flow by Laser-Doppler technique and hemoglobin oxygenation and hemoglobin concentration in tissue by spectrometric techniques, has been described in detail elsewhere [14]. The local oxygen supply parameters, blood flow, oxygen saturation of hemoglobin, and the relative postcapillary venous filling pressures were recorded by an optical fiber probe. The fiber probe incorporates both the laser Doppler method and the broadband light spectrometry technique. The probe we used simultaneously assessed data in 2 millimeters as a superficial tissue depth and 8 millimeters as a deep tissue depth of the free flap regarding:

- capillary blood flow [arbitrary units AU]

- skin oxygen saturation [\%]

- relative postcapillary venous filling pressure [AU]

Laser doppler flowmetry: The tissue is illuminated with coherent laser light and backscattered light is collected by the same probe. Frequency shifted light is extracted by heterodyne light beating technique. As laser Doppler flowmetry detects all moved particles of certain velocity, it measures blood flow. We have recently described the use of the $\mathrm{O} 2 \mathrm{C}$ system in measurements of increased capillary blood flow at the point of pain in patients with insertional and mid-portion tendinopathy of the Achilles tendon compared to healthy subjects as well as the influence of permanent and intermittent application of cooling and compression on microcirculation of Achilles tendons in healthy humans [15,16].

Regarding the intraobserver reliability of the laser Doppler in the $\mathrm{O} 2 \mathrm{C}$-system, a $5 \%$ intrasubject variability was determined indicating the $\mathrm{O} 2 \mathrm{C}$ system as a reliable method under sufficient standardized test conditions [17]. Microcirculatory data assessment using the O2C-system as well as temperature assessment was performed by the same experienced examiner. Data was blinded for statistical analysis and performed by another examiner.

\section{Statistics}

Data assessment was performed by the same experienced investigator and blinded regarding location of measurement and flap type before statistical analysis was performed by a statistician. We conducted a Pearson Correlation as well as an independent samples t-test between free flap microcirculation and flap skin temperature. A p-value less than 0.05 was considered to indicate statistical significance. The SPSS-statistical- 
software-package 16.0 for Windows (SPSS Inc, Chicago, Ill, USA) was used for statistical analysis.

\section{Results}

\section{Participants}

A total number of 56 patients ( 35 males, 21 females) were enrolled in this clinical trial after free flap soft-tissue defect coverage between November 2009 and January 2011.

Mean patient's age was $49 \pm 14 y$ rs. The free flaps were transferred either to the extremities, to the head or to the chest for either soft tissue or breast reconstruction. Eleven patients received soft tissue coverage due to a soft tissue defect from an open tibia fracture, eight patients due to decollement injury, four patients due to postoperative wound infection as tertiary wound closure after normalization of inflammation parameters, three patients due to forearm amputation, six patients due to mamma carcinoma metastasis resection, twelve patients due to burn injury and two patients due to chronic crural ulceration. Ten female patients underwent prior breast resection due to breast cancer.

Between November 2009 and January 2011 twenty-four latissimus dorsi flaps, one serratus fascia flap, nine deep inferior epigastric perforator (DIEP) flaps, one vertical rectus abdominis muscle (VRAM) flap, eleven anterolateral thigh (ALT) flaps and ten parascapular flaps were transplanted, enrolled in the study and monitored postoperatively up to postoperative day seven using combined Laser-Doppler and photospectrometry as well as temperature monitoring.

Of all transplanted free flaps twenty-four were transplanted to the lower extremity, fourteen were transplanted to the upper extremity, thirteen were transplanted for breast or chest reconstruction and finally six flaps were transplanted to the head. We did not perform AV-Loop surgery in any of the patients.

The history of the patients showed coronary artery disease with former myocardial infarction in four patients. Sixteen patients suffered from breast cancer. Two patients had osteoporosis and hypothyreosis. Two patients suffered from a postthrombotic syndrome. Five patients had a positive history of multiple sclerosis.

\section{Microcirculatory monitoring}

We found that mean deep microcirculatory blood flow of all assessed free flaps was 109 \pm 37 arbitrary units [AU]. Adjacent skin mean capillary blood flow was $143 \pm 52 \mathrm{AU}$. Mean free flap skin island temperature was $95.3 \pm 3.0^{\circ} \mathrm{F}$. Mean temperature of the adjacent healthy skin was $96.6 \pm 1.9^{\circ} \mathrm{F}$.

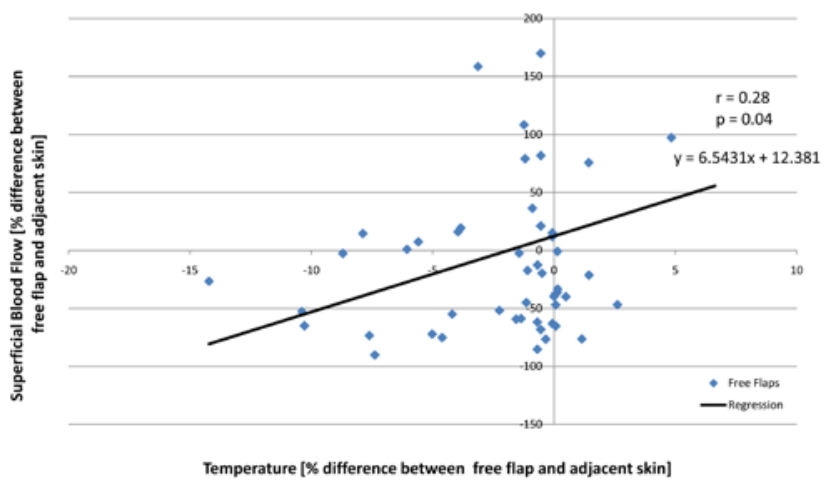

Figure 1: Superficial Blood Flow - Temperature - Correlation.

\begin{tabular}{|l|c|c|c|c|}
\hline & \multicolumn{2}{|c|}{ Superficial tissue depth } & \multicolumn{2}{c|}{ Deep tissue depth } \\
\cline { 2 - 5 } & Free flap skin & $\begin{array}{c}\text { Adjacent } \\
\text { healthy skin }\end{array}$ & Free flap skin & $\begin{array}{c}\text { Adjacent } \\
\text { healthy skin }\end{array}$ \\
\hline $\begin{array}{l}\text { Skin tempera- } \\
\text { ture [ }{ }^{\circ} \text { F] }\end{array}$ & $95.3 \pm 3.0$ & $96.6 \pm 1.9$ & $95.3 \pm 3.0$ & $96.6 \pm 1.9$ \\
\hline $\begin{array}{l}\text { Skin oxygen } \\
\text { saturation [\%] }\end{array}$ & $44.9 \pm 19.6$ & $46.7 \pm 17.9$ & $46.0 \pm 18.7$ & $49.2 \pm 17.5$ \\
\hline $\begin{array}{l}\text { Relative } \\
\text { postcapillary } \\
\text { venous filling } \\
\text { pressure [AU] }\end{array}$ & $25.0 \pm 12.6$ & $25.9 \pm 10.5$ & $54.3 \pm 17.2$ & $50.4 \pm 14.1$ \\
\hline $\begin{array}{l}\text { Capillary blood } \\
\text { flow [AU] }\end{array}$ & $16.7 \pm 13.9$ & $22.7 \pm 16.4$ & $108.8 \pm 36.9$ & $143.2 \pm 52.1$ \\
\hline
\end{tabular}

Table 1: Absolute values (mean $\pm S D$ ) of skin temperature, skin oxygen saturation, postcapillary venous filling pressure and capillary blood flow of the free flap skin island and the adjacent healthy skin in a superficial tissue depth $(2 \mathrm{~mm})$ and deep tissue depth $(8 \mathrm{~mm})$. Temperature assessment was the same for superficial and deep tissue depth.

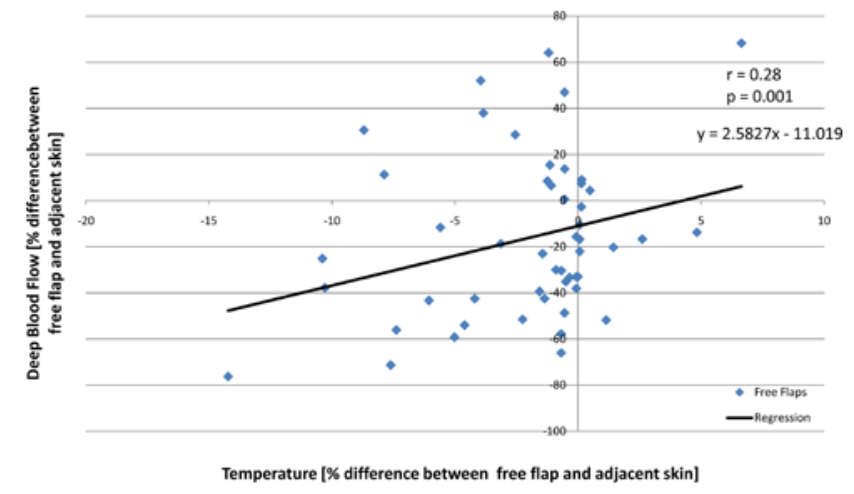

Figure 2: Deep Blood Flow - Temperature - Correlation.

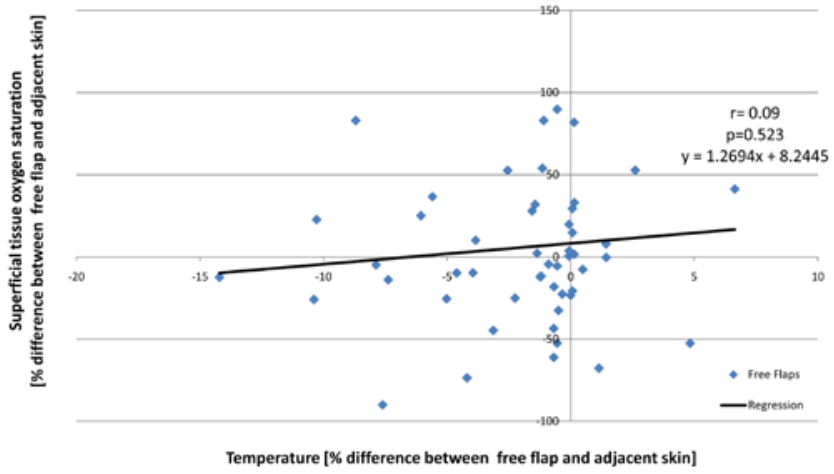

Figure 3: Superficial tissue oxygen saturation - Temperature - Correlation.

We found a positive correlation between the percentaged difference between free flap and adjacent skin temperatures and percentaged difference of free flap and adjacent skin capillary blood flow (Pearson Correlation $\mathrm{r}=0.28$, $\mathrm{p}=0.001$ ) in 56 free flaps (Figure 1). An overview about the mean absolute values of temperature and microcirculatory parameters of the free flap skin island and the healthy adjacent skin both in the superficial tissue depth $(2 \mathrm{~mm})$ and the deep tissue depth $(8 \mathrm{~mm})$ are demonstrated in Table 1.

\section{Clinical free flap failures}

Onefreeflap showed temperature drop to $78.8^{\circ} \mathrm{F}$ caused by postoperative combined arterial and venous thrombosis. Simultaneous microcirculatory assessment of free flap blood flow on this flap demonstrated a reduction 


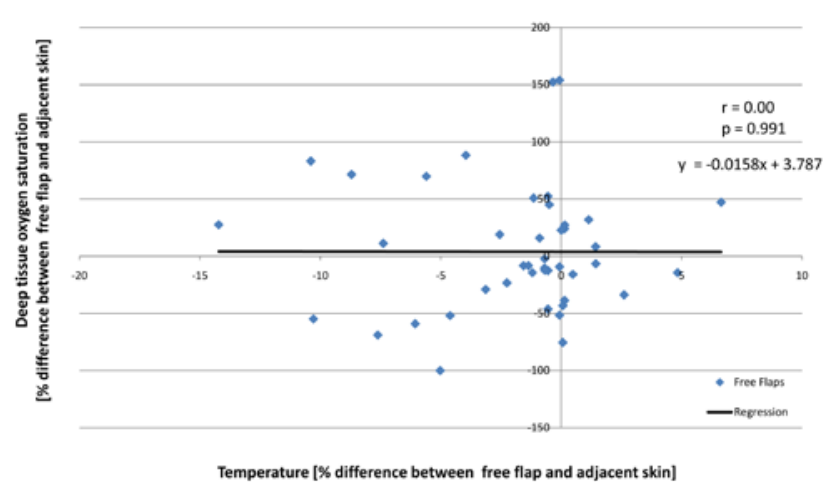

Figure 4: Deep tissue oxygen saturation - Temperature - Correlation.

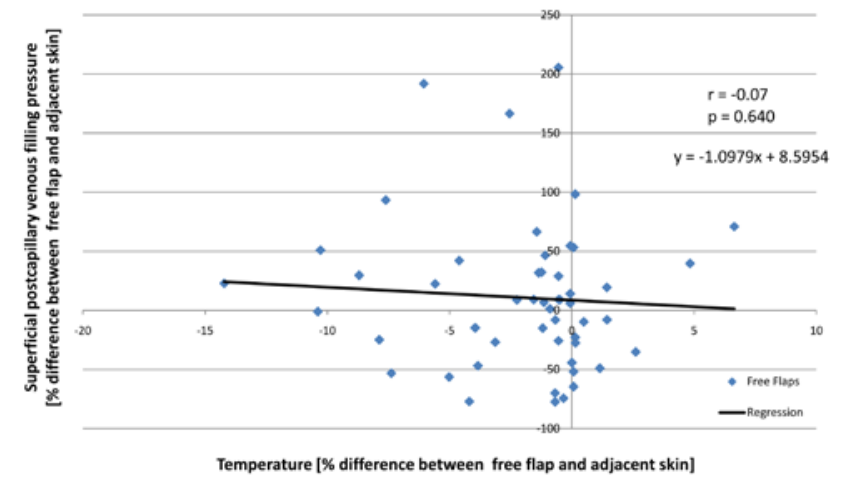

Figure 5: Superficial postcapillary venous filling pressure - Temperature - Correlation.

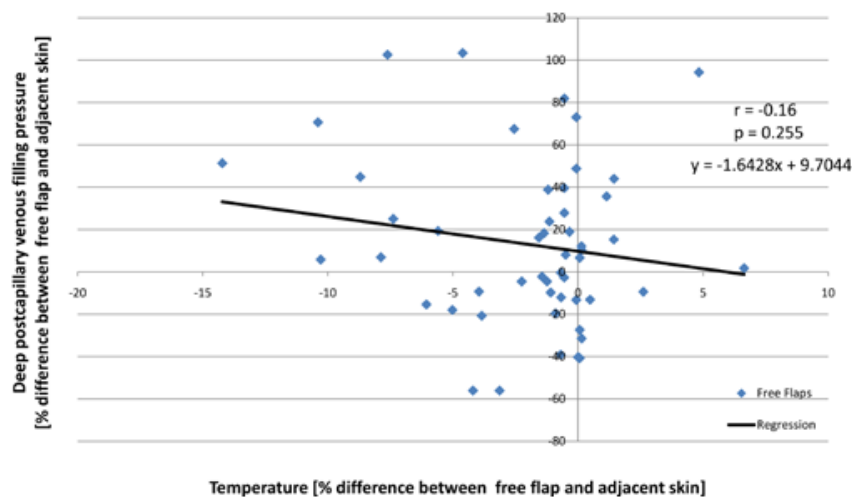

Figure 6: Deep postcapillary venous filling pressure - Temperature - Correlation.

in microcirculatory blood flow to 16AU. Consecutive revision of the arterial and venous anastomosis with thrombectomy was performed and final flap survival achieved. Postoperative flap temperature with regular microcirculatory blood flow was $97.2^{\circ} \mathrm{F}$.

\section{Microcirculatory blood flow}

Percentage superficial and deep blood flow difference between free flap skin island and adjacent skin significantly correlated to percentaged temperature difference between free flap and adjacent skin $(r=0.28 ; p=0.04$ and $\mathrm{p}=0.001$, respectively; (Figure 1,2 ). A reduction of $10 \%$ free flap skin island temperature showed a decrease of the mean superficial and deep free flap microcirculatory capillary blood flow by $50 \%$ and $40 \%$, respectively.

\section{Superficial and deep skin oxygen saturation}

Percentage difference of superficial and deep skin oxygen saturation in $2 \mathrm{~mm}$ and $8 \mathrm{~mm}$ tissue depths did not show a significant correlation to temperature assessment $(\mathrm{r}=0.09, \mathrm{p}=0.52 ; \mathrm{r}=0.00, \mathrm{p}=0.99)$.

\section{Relative post capillary venous filling pressure}

Percentage difference between free flap and adjacent skin of superficial and deep relative postcapillary venous filling pressure in $2 \mathrm{~mm}$ and $8 \mathrm{~mm}$ tissue depth did not correlate significantly to percentaged difference between free flap and adjacent skin free flap temperature $(r=-0.07, p=0.640$; $\mathrm{r}=-0.16, \mathrm{p}=0.255$, respectively).

\section{Discussion}

The aim of this study was to evaluate the relationship of free flap skin island and healthy adjacent skin temperatures to microcirculatory hemodynamics in clinical free flap transplantation in order to eliminate or at least potentially minimize the environmental influences on temperature assessment as a noninvasive free flap monitoring option. We therefore hypothesized that the difference between free flap skin island and healthy adjacent skin microcirculation correlates to the difference between free flap skin island and healthy adjacent skin temperatures. Based on our prospective cohort clinical study registered at ClinicalTrials.gov our hypothesis was confirmed.

We could demonstrate that the difference between free flap skin island temperature and healthy adjacent skin temperature is related to the microcirculatory capillary blood flow of a given free flap. The lower the free flap skin island temperature was compared to the adjacent skin temperature, the worse capillary blood flow was apparent throughout the free flap. A 10\% reduction of free flap skin island temperature compared to adjacent skin indicated a deterioration of flap microcirculation by $50 \%$, if temperature of the adjacent healthy skin remained constant. This should be discussed in detail.

Free flap monitoring has become an important issue in Plastic and Reconstructive Surgery. If occlusion of arterial inflow or venous outflow occurs, a free flap can still be successfully salvaged by immediate reexploration and revision of the microanastomoses. The success of such secondary microsurgery is inversely related to the time interval between the onset of flap ischemia and its clinical recognition [18]. Hanasono et al. were among the the first to quantify changes in venous blood velocity after free tissue transfer. They showed that flow velocity increases over the first 3 days and may decrease slightly thereafter [19].

Various new and innovative free flap monitoring techniques have been developed, but none of these techniques have yet succeeded to meet ideal criteria. Recently, different monitoring systems were meta-analyzed [20]. The implantable Doppler system, near-infrared spectroscopy, and laser Doppler flowmetry appeared to be the best monitoring devices currently available. By using combined laser Doppler flowmetry and spectrophotometry simultaneously to free flap temperature assessment, we recently demonstrated that free flap skin temperature is closely related to free flap microcirculation [12].

The lack of clear cut values to predict flap failure, technical difficulties with temperature measuring probes and especially concerns regarding the effect of environmental conditions on flap temperature have contributed to the lack of uniform acceptance of temperature measurement as a reliable method of postoperative flap monitoring. On the other hand, when properly applied and interpreted, the sensitivity of surface-temperature recording has already been shown to be as high as 98 percent including a predictive 
value of 75 percent, making it a simple, inexpensive, and highly reliable technique of free-flap monitoring [21]. Papillion et al. recently described a difference between flap temperature and adjacent skin temperatures in two groups of free flaps. Differences in temperature between survival and failure groups were evaluated for statistical significance. In a $24 \mathrm{~h}$ postoperative period, the flap failure group had a higher difference between its temperature and the adjacent skin control temperature compared with a lower temperature difference in the surviving flaps and their adjacent skin control.

According to that and our own study, we postulated that linear continuous temperature assessment might be more reliable if environmental effects as mainly cooling of the flap would be less misinterpreted as impaired flap's microcirculation if the temperature of the flap could be set in contrast to the regular perfused adjacent healthy skin with also cooling from environmental issues and not from impaired microcirculation caused by flap failure. Contributing to that, thermoregulatory blood flow is regulated primarily by the sympathetic nervous system on arteriovenous anastomoses, which shunt blood directly from the arterioles to the venules to dissipate heat by reduction of skin or extremity perfusion when core temperature decreases or when the extremity is exposed to cold. Free flaps are sympathectomized which may reduce vascular tone and improve microcirculation, as demonstrated in animal works or in free flap surgery resulting in less temperature reduction as in healthy adjacent skin [22,23]. Therefore, environmental factors like air temperature around the flap might not influence the free flap's perfusion as much as it influences healthy skin where cutaneous microcirculation is reduced if the skin is exposed to cold air in non-acral skin. Consequently, free flap skin temperature should remain on the same level as the adjacent healthy skin as long as microcirculation is not impaired. Recently, Sun et al. demonstrated that simultaneous denervation and revascularization of free forearm flaps increased the regional blood flow significantly which was associated with the sympathetic denervation and reactive hyperemia, respectively [24]. Patients after sympathectomy have been found to suffer from severe hypoadrenergic orthostatic hypotension as a result from sympathetic deregulation [25]. This finding could also contribute to an increased free flap temperature when the flap is set under orthostatic stress with higher arterial inflow. On the other hand, under orthostatic stress deteriorated venous outflow could result in impaired flap perfusion with decreased flap temperature as demonstrated in our recent study. Nevertheless, comparing differences between the sympathectomized free flap skin island temperature and adjacent skin temperature might be more reliable if the differences of microcirculatory hemodynamics of free flaps and adjacent skin correlate in an equivalent way.

Interestingly, our study found free flap capillary blood flow correlating to the percentaged difference between free flap skin island and healthy adjacent skin temperatures, which has never been demonstrated before. In addition we could demonstrate a linear regression between free flap microcirculation and skin temperatures. A percentaged temperature difference of $10 \%$ correlates to a free flap capillary blood flow reduction by approximately $50 \%$.

The final decision to explore a microvascular anastomosis is usually based on clinical examination, but the trigger to clinically examine a potentially compromised free flap may be based on easily and straightforward obtained surface temperature measurements.

\section{Limitations}

This study enrolled 56 free flaps with a complication rate in terms of flap revision of $2 \%$. Although, we even found a significant correlation between differences of free flap to adjacent skin perfusion and free flap and adjacent skin temperatures in the majority of regular perfused free flaps, further studies have to evaluate the correlation of the aforementioned parameters in emergent flap failure with documentation of temperature and microcirculation over time until flap salvage or flap loss.

Furthermore, different free flaps, e.g. latissimus dorsi flaps, anterolateral thigh flaps or deep inferior epigastric flaps transplanted to different locations, e.g. lower limb, breast or the head might have different microcirculation. Consequently, a microcirculatory mapping of free flaps at different locations of the body with simultaneous temperature assessment must be initiated to establish more valid data for free flap monitoring. In our study, blinding of the different free flaps and data assessment locations was performed before statistical analysis in order to evaluate the correlation of temperature and microcirculation of free flap and adjacent healthy skin in general for our most often used free flaps. Thorough investigation has to be performed in the future for all free flap monitoring devices, if there are differences in liability in different free flaps.

\section{Conclusion}

Our study demonstrates a relationship between differences of free flap Skin Island and adjacent skin temperatures to microcirculation of a given free flap. Our primary hypothesis was confirmed. As 10\% less free flap skin island temperature compared to adjacent skin, if skin temperature of the adjacent skin remains on the same level, shows a decrease of free flap microcirculation by $50 \%$ and according to recent studies, we postulate an acute temperature reduction of 3 degrees Fahrenheit or more at the center of the skin island as indicative of arterial thrombosis, whereas a reduction of $1^{\circ} \mathrm{C}$ to $2^{\circ}$ Fahrenheit uniform over of the free flap skin island could be indicative of venous compromise, if skin temperature of the adjacent skin remains constant. Eliminating environmental influences on temperature assessment by comparing the temperature of the skin island of the free flap to the temperature of the adjacent healthy skin could make free flap temperature monitoring more reliable in reconstructive microsurgery in the future. However, randomized-controlled trials are pending to elucidate the value of clinical temperature monitoring following reconstructive microsurgery.

\section{References}

1. Siemionow M, Arslan E (2004) Ischemia/reperfusion injury: A review in relation to free tissue transfers. Microsurgery 24: 468-475.

2. Khouri RK, Cooley BC, Kunselman AR, Landis JR, Yeramian P, et al. (1998) Prospective study of microvascular free-flap surgery and outcome. Plast Reconstr Surg 102: 711.

3. Bui DT, Cordeiro PG, Hu QY, Disa JJ, Pusic A et al. (2007) Free flap reexploration: Indications, treatment, and outcomes in 1193 free flaps. Plast Reconstr Surg 119: 2092.

4. Salgado CJ, Smith A, Kim S, Higgins J, Behnam A, et al. (2002) Effects of late loss of arterial inflow on free flap survival. J Reconstr Microsurg 18: 579-584.

5. Brown JS, Devine JC, Magennis P, Sillifant P, Rogers SN, et al. (2003) Factors that influence the outcome of salvage in free tissue transfer. $\mathrm{Br} \mathrm{J}$ Oral Maxillofac Surg 41: $16-20$

6. Khouri RK, Shaw WW (1992) Monitoring of free flaps with surfacetemperature recordings: Is it reliable? Plast Reconstr Surg 89: 495-9.

7. Acland RD. Discussion (1981) Experience in monitoring the circulation in free-flap transfers. Plast Reconstr Surg 68: 543-55.

8. Busic V, Das-Gupta R (2004) Temperature monitoring in free flap surgery. $\mathrm{Br}$ Plast Surg 57: 588.

9. Bulstrode NW, Wilson GR, Inglis MS (2002) No-touch free-flap temperature monitoring. Br J Plast Surg 55: 174.

10. Papillion P, Wong L, Waldrop J, Sargent L, Brzezienski M, et al. (2009) Infrared surface temperature monitoring in the postoperative management of free tissue transfers.Can J Plast Surg Fall 17: 97-101. 
Citation: Kraemer R, Lorenzen J, Wuerfel W, Papst S, Busche M, et al. (2011) Elimination of Environmental Effects on Temperature Assessment in Clinical Postoperative Free Flap Monitoring - A Prospective Cohort Study. Anaplastology S1:001. doi:10.4172/2161-1173.S1-001

11. Chiu ES, Altman A, Allen RJ Jr, Allen RJ Sr, (2008) Free flap monitoring using skin temperature strip indicators: adjunct to clinical examination. Plast Reconstr Surg 122: $144 \mathrm{e}-145 \mathrm{e}$.

12. Kraemer R, Lorenzen J, Knobloch K, Papst S, Kabbani M, et al. (2011) Free flap microcirculatory monitoring correlates to free flap temperature assessment. J Plast Reconstr Aesthet Surg. 64: 1353-8.

13. Bossuyt PM, Reitsma JB, Bruns DE, Gatsonis CA, Glasziou PP, et al. (2003) Standards for Reporting of Diagnostic Accuracy.The STARD statement for reporting studies of diagnostic accuracy: explanation and elaboration.Ann Intern Med 138: W1-12.

14. Frank KH, Kessler M, Appelaum K, Dümmler W (1989) The erlangen microlightguide spectrophotometer EMPHO I. Phys Med Biol 34: 1883-1900.

15. Knobloch K, Kraemer R, Lichtenberg A, Jagodzinski M, Gossling T, et al. (2006) Achilles tendon and paratendon microcirculation in mid-portion and insertional tendinopathy in sportsmen. Am J Sports Med 34: 92-7.

16. Knobloch K, Kraemer R, Lichtenberg M, Gossling T, Zeichen J, et al. (2006) Microcirculation of the ankle after Cryo/Cuff application in healthy volunteers. Int J Sport Med 27: 250-5.

17. Ghazanfari M, Vogt L, Banzer W, Rhodius U (2002) Reproducibility of non-invasive blood flow measurements using laser Doppler spectroscopy. Phys Med Rehab Kuror 12: 330-336.
18. Goodstein WA, Buncke HJ (1979) Patterns of vascular anastomosis vs. success of free groin flap transfers. Plast Reconstr Surg. 64: 37-40.

19. Hanasono MM, Ogunleye O, Yang JS, Hartley CJ, Miller MJ (2009) Changes in blood velocity following microvascular free tissue transfer. J Reconstr Microsurg 25:417-24. Epub 2009 Jul 10.

20. Salgado CJ, Moran SL, Mardini S (2009) Flap monitoring and patient management Plast Reconstr Surg 124: e295-302.

21. Khouri RK, Shaw WW (1992) Monitoring of free flaps with surface temperature recordings: Is it reliable? Plast Reconstr Surg 89: 495.

22. Pollock DC, Li Z, Rosencrance E, Krome J, Koman LA, et al. (1997) Acute effects of periarterial sympathectomy on the cutaneous microcirculation. J Orthop Res 15 $408-413$.

23. Lorenzetti F, Ahovuo J, Suominen S, Salmi A, Asko-Seljavaara S (2002) Color Doppler ultrasound evaluation of hemodynamic changes in free tram flaps and their donor sites. Scand J Plast Reconstr Surg Hand Surg 36: 202-206.

24. Sun TB, Kuo TB, Yang CC (2009) Power spectral analysis of perfusion signals on free radial forearm flap transplantation in humans. Microsurgery 29: 636-43.

25. Van Lieshout JJ, Wieling W, Wesseling KH, Endert E, Karemaker JM (1990) Orthostatic hypotension caused by sympathectomies performed for hyperhidrosis. Neth J Med 36: 53-7.
This article was originally published in a special issue, Reconstructive Surgery handled by Editor(s). Dr. Karsten Knobloch, Hannover Medical School, Germany; Dr. Sammy Al-Benna, Ruhr University Bochum, Germany; Dr. Namdar Thomas, Ruhr University Bochum, Germany 